\title{
Glycine-Rich Proteins Encoded by a Nodule-Specific Gene Family Are Implicated in Different Stages of Symbiotic Nodule Development in Medicago Spp.
}

\author{
Zoltán Kevei, ${ }^{1,2}$ José María Vinardell, ${ }^{1}$ György B. Kiss, ${ }^{2}$ Adam Kondorosi, ${ }^{1,2}$ and Eva Kondorosi ${ }^{1}$ \\ ${ }^{1}$ Institut des Sciences du Végétal, CNRS UPR 2355, Avenue de la Terrasse, 91198 Gif-sur-Yvette, France; ${ }^{2}$ Institute \\ of Genetics, Biological Research Center, Hungarian Academy of Sciences, 6701 Szeged, Hungary
}

Submitted 17 December 2001. Accepted 23 May 2002.

Four genes encoding small proteins with significantly high glycine content have been identified from root nodules of Medicago sativa. All of these proteins as well as their Medicago truncatula homologues carried an amino terminal signal peptide and a glycine-rich carboxy terminal domain. All except nodGRP3 lacked the characteristic repeat structure described for cell wall and stress response-related glycine-rich proteins (GRP). Expression of these GRP genes was undetectable in flower, leaf, stem, and hypocotyl cells, whereas expression was highly induced during root nodule development, suggesting that $G R P$ genes act as nodulins. Moreover, none of these nodule-expressed GRP genes were activated by hormones or stress treatments, which are inducers of many other GRPs. In Rhizobium-free spontaneous nodules and in nodules induced by a noninfective mutant strain of Sinorhizobium meliloti, all these genes were repressed, while they were induced in $\mathrm{Fix}^{-}$nodules, unaffected in bacterial infection, but halted in bacteroid differentiation. These results demonstrated that bacterial infection but not bacteroid differentiation is required for the induction of the nodule-specific GRP genes. Differences in kinetics and localization of gene activation as well as in the primary structure of proteins suggest nonredundant roles for these GRPs in nodule organogenesis.

Additional keyword: leguminous plants.

Glycine-rich proteins (GRP) exhibit high structural diversity in plants. This large gene family is characterized by quasi-repetitive glycine-rich domains, most frequently with GGGX, GGXXXGG or GXGX repeats, resulting in proteins of distinct sizes (Sachetto-Martins et al. 2000). In most proteins, amino terminal signal peptides as well as other specific structures, such as cold shock domain (Kingsley and Palis 1994), cysteinrich pattern (Rohde et al. 1990), or RNA-binding motifs (RNP1 and RNP-2) (Dreyfuss et al. 1988) have also been identified.

The diverse expression pattern and subcellular localization of various GRPs suggest their implication in different physiological processes. Most GRPs are developmentally regulated

Corresponding author: E. Kondorosi; E-mail: Eva.Kondorosi@isv.cnrsgif.fr.

Current address for J. M. Vinardell: Departamento de Microbiologia, Facultad de Biologia, Universidad de Sevilla, avda. Reina Mercedes, 41012 Sevilla, Spain. and induced by biotic and abiotic factors. Salicylic acid (Hammond-Kosack and Jones 1996), abcisic acid (de Oliveira et al. 1990), methyl jasmonate (Molina et al. 1997), or ethylene induce many GRP genes (Memelink et al. 1990), while auxin has a negative effect on the expression of certain GRPs (Reddy and Poovaiah 1987). Pathogenic attacks such as viral (Linthorst et al. 1990) or fungal infections (Molina et al. 1997) also modulate GRP expression. Root knot nematode Meloidogyne incognita infection in alfalfa results in the induction of a GRP possessing an RNA-binding motif (Potenza et al. 2001).

Among the abiotic factors, regulation of GRPs can be elicited by osmotic stress (Gómez et al. 1988; Xu et al. 1995). Cold shock (Carpenter et al. 1994) and wounding (Showalter et al. 1991) activate GRP genes, while light (Kaldenhoff and Richter 1989) and circadian rhythm modulate GRP expression (Cretin and Puigdomenech 1990).

At the subcellular level, many GRPs were localized in the cell wall or were membrane-associated, but they were found also in the nucleus and cytosol (Sachetto-Martins et al. 2000). Organand tissue-specific expression studies showed the presence of $G R P$ transcripts in the protoxylem (Keller et al. 1989), xylem, (Harrak et al. 1999) and phloem, as well as in epidermal tissues (Condit 1993). Moreover, anther-specific GRPs were identified from flowers (Mousavi et al. 1999), while other GRPs were detected during fruit development (Santino et al. 1997).

Based on their cell wall localization, several highly expressed GRP were reported as structural components of the plant cell wall, similar to proline-rich or hydroxyproline-rich proteins (Cassab 1998). Various studies on the regulation of the $G R P$ genes suggest multiple roles in plant development. Functional studies revealed that GRPs might be implicated in cell lignification (Condit 1993) and mediating membrane-cytoskeleton or membrane-cell wall interconnections (Marty et al. 1996). GRPs with RNA-binding motifs might be involved in RNA processing or in the control of gene expression (Heintzen et al. 1994). Other GRP may be implicated in the stabilization of lipid-containing structures (Ross and Murphy 1996). So far, only the Arabidopsis thaliana AtGRP3 function has been demonstrated. This protein plays a role in the WAK1 (wall-associated receptor kinase) signaling pathway, where its binding to the WAK1 receptor kinase is necessary for the activation of KAPP, a kinase-associated phosphatase (Anderson et al. 2001; Park et al. 2001).

GRPs were also isolated from nitrogen-fixing root nodules of Vicia faba (Küster et al. 1995; Schröder et al. 1997). This unique plant organ develops in leguminous plants under com- 
bined nitrogen limitation in a symbiotic interaction with the rhizobial partner. Bacterial signal molecules (Nod factors) are required for nodule induction and different surface polysaccharides (EPS, KPS, and LPS) are involved in the invasion of plant cells. The indeterminate nodules developing in temporal legumes contain a persistent apical meristem (zone I), an invasion zone (zone II), in which cells arrested in division become infected with rhizobia and differentiate gradually along a few cell layers to reach the terminal phase for nitrogen fixation in zone III. In addition to housekeeping genes, consecutive activation of specific gene sets producing nodule-specific plant proteins (nodulins) are required for the various stages of nodule development.

Nodulin genes are classified as early and late nodulins, depending whether their expressions precede or accompany nitrogen fixation (Crespi and Gálvez 2000; Schultze and Kondorosi 1998). The GRP genes isolated from Vicia faba (Vfnod-GRPI, Vfenod-GRP2, Vfenod-GRP3, Vfnod-GRP4, and Vfenod-GRP5) exhibited nodule-specific expression, and tissue print hybridization localized their transcripts, mainly in the interzone II-III and the nitrogen-fixing zone III of the indeterminate nodules. Nodulin 24, a constituent of the peribacteroid membrane in soybean (Cheon et al. 1994) was also classified as GRP. In the nitrogen-fixing symbiosis between Frankia and the actinorhizal plant Alnus glutinosa, nodule-specific GRP were also detected in the cortical cells of infected nodules (Pawlowski et al. 1997). In Medicago spp., NMs22 (Ganter et al. 1998) appears to encode a nodule-specific alfalfa glycine-rich protein. Screenings and sequencing of Medicago cDNA libraries in our laboratory also resulted in the identification of homologues of the broad bean Vfenod-GRP5 from M. sativa (Jiménez-Zurdo et al. 2000) and from M. truncatula (Györgyey et al. 2000).

Our goal in this work was to isolate GRP genes from $M$. sativa and $M$. truncatula nodules and to get an insight into their function during nodule development. We addressed the following questions. i) Is the expression of the GRP genes nodule specific? ii) What are the requirements and signals for gene activation? iii) At what stage or stages of nodule development are the genes switched on? iv) Are the gene expressions specific for the different nodule zones or cell layers?

Our study revealed that all the isolated GRPs expressed exclusively in the nodules and encoded small proteins with a hydrophobic signal peptide that shared no homology with other GRPs in the databases. They represented nodulins as well as a novel subfamily of GRP that showed structural similarity only to Vicia GRP nodulins. The studied GRP genes were induced at different time points after inoculation and also exhibited differences in the kinetics and localization of gene expression. Moreover, we demonstrated that expression of these GRP genes was dependent on the infection of nodule cells by the microsymbiont Sinorhizobium meliloti but was not induced by abiotic or biotic factors. Analysis of the kinetics of gene expression and the localization of their transcriptions in nodules suggests that the nodule-specific GRPs might play nonredundant roles required at specific stages of nodule development.

\section{RESULTS}

\section{Identification of cDNAs coding for GRP from Medicago nodules.}

For the isolation of genes encoding GRP, a cDNA library made of young nodules of Medicago sativa subsp. varia A2 was hybridized with the V. faba Vfnod-GRP1, Vfenod-GRP2, Vfenod-GRP3, Vfnod-GRP4, or Vfenod-GRP5 cDNA clones (Schröder et al. 1997) under low stringency to obtain both orthologue and related genes. These screenings resulted in the isolation of 37 cDNAs that represented four distinct genes en- coding GRP. MsnodGRP1 (accession number AF498986), represented by 11 cDNAs, was isolated with the Vfnod-GRPI probe that displayed limited homology (66\% identity at nucleic acid level) with the probe. Screening with Vfnod-GRP4 led to the isolation of 16 clones corresponding to two types of cDNAs; MsnodGRP2A and MsnodGRP2B (accession numbers AF498987 and AF498988, respectively) that were highly homologous to each other but distinct from Vfnod-GRP4. The MsnodGRP3 (accession number AF498989) cDNAs (10 clones) were obtained with Vfenod-GRP5 and exhibited $72 \%$ identity with the probe. In some cases due to the low stringency of hybridization, the screening also resulted in the isolation of non-nodule specific GRPs, like the homologue of polyadenylate-binding protein (Le et al. 1997) isolated from several plants and the recently described alfalfa GRP induced by root knot nematode (Potenza et al. 2001), which were not, however, studied further.

The MsnodGRP1, MsnodGRP2A, and MsnodGRP2B cDNA clones were partial. A homology search in the $M$. truncatula expressed sequence tag (EST) database has led, however, to the identification of the full-length sequences of these clones. All of these cDNAs coded for small putative proteins. MsnodGRP1 was 112, MsnodGRP2A was 101, MsnodGRP2B was 114, and MsnodGRP3 was 217 amino acids long (Fig. 1). All of them possessed a putative hydrophobic amino terminal secretory signal peptide predicted by the algorithm of von Heijne (1986) and a glycine-rich carboxy terminal part. Although, glycine-rich repeats are the major structural features of GRPs, in the MsnodGRP1, MsnodGRP2A, and MsnodGRP2B gene products, no glycine-rich repetitive blocks were present, in contrast to MsnodGRP3, which contained four repeats of 42, 40, 42, and 42 amino acids. Within these repeats, a WRDWG-
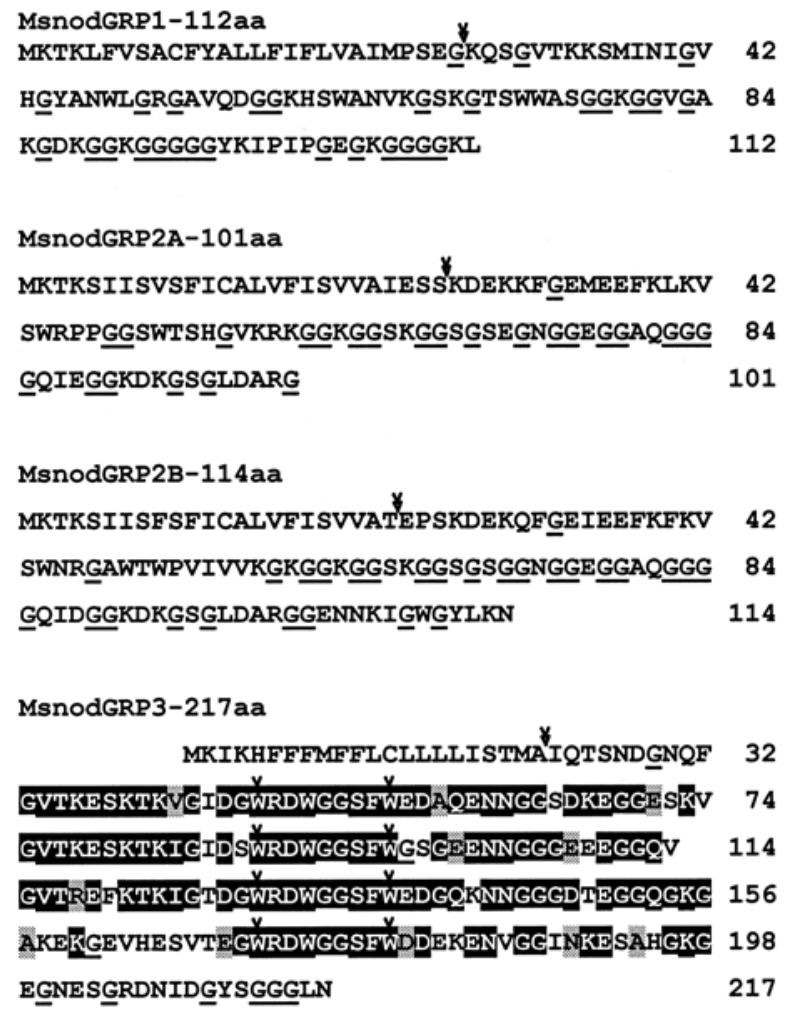

Fig. 1. Primary structure of glycine-rich proteins from Medicago sativa nodules. Glycine residues are in bold; signal peptides are underlined. In the four repeats of MsnodGRP3 (33-74, 75-114, 115-156, and 157-198), identical amino acid residues present in at least two repeats are on black background, while similar amino acids are shown on gray. The start and the end of WRDWGGSFW motifs are indicated with vertical arrows. 
GSFW oligopeptide sequence was conserved (Fig. 1). An orthologue of the MsnodGRP3 cDNA, MtnodGRP3 (accession number AF498995), was found also in M. truncatula by systematic sequencing of a nodule cDNA library (MtNo388) in our laboratory (Györgyey et al. 2000). The MtnodGRP3 protein displayed $94 \%$ similarity and $88 \%$ identity to
MsnodGRP3. The molecular structure of MsnodGRP3 resembled to that of NMs22 from alfalfa and that of Vfenod-GRP5 (Fig. 2A). Although the sizes of these proteins were different, all of them carried an $\mathrm{N}$-terminal signal peptide and downstream repetitive glycine-rich blocks. In contrast to the four repeats in MsnodGRP3, NMs22 contained three repeats with

A

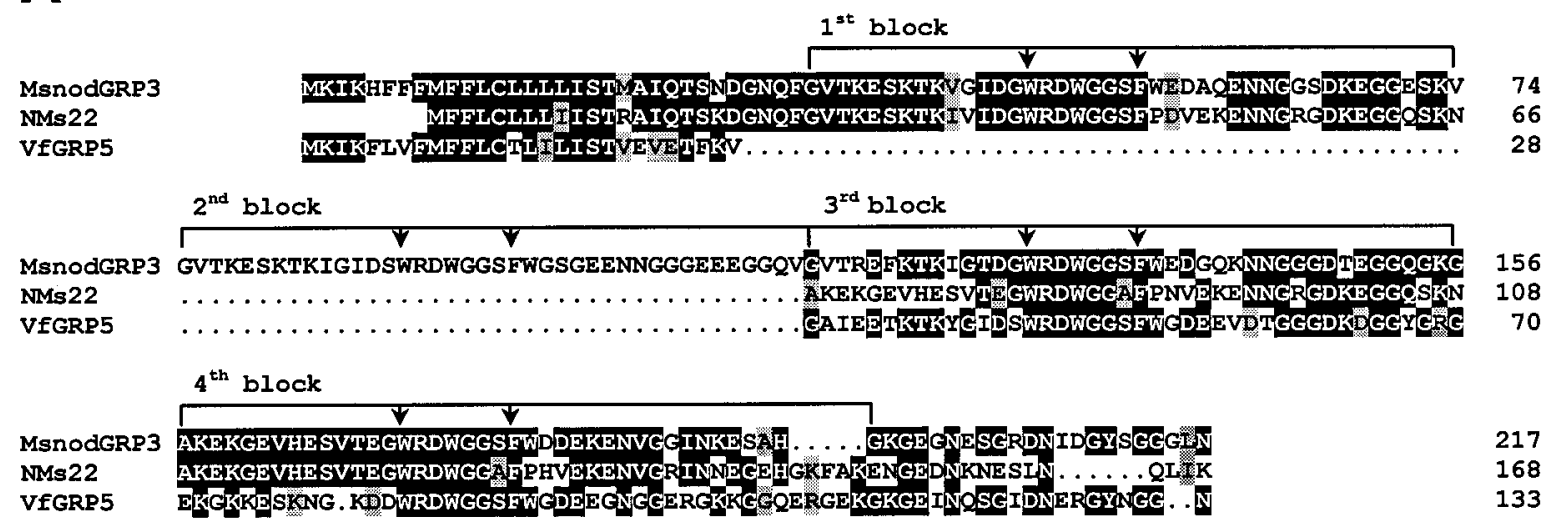

$\mathbf{B}$

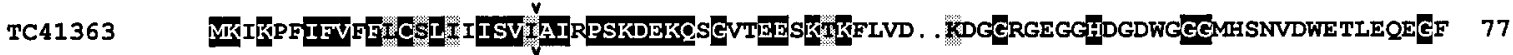
MEN29 MKTKPUIFVFGICPLIIISV AIIRSSK. . . VGETENSKNWIGVE . . RVEDEHNGDFEGGNVDGLFENIIHRMVRLIG 73 MsnOdGRP2B MKTKSIISFSEICALVEISVVA TEPSKDEKQFGEIEEFKEK. . . VSWRNGAWWWVIVVKGKGGKGGSKGGSGSGGNGG 76 MtnOdGRP2B MKTKSIISFFEVCAIYFISV AATEPSKDEKQFGEIEEFKSK. . . VSWNRGAWWWKYGVKGNGGKGGSKGGSGSGENGG 76 MSnOdGRP2A MKTKSIISVISFICALVEISWAIESSKDEKKFGEM FEFKLK . . VSWRPPGESWTTSEGVKRKGGKGGSKGGSGSEGNGE 76

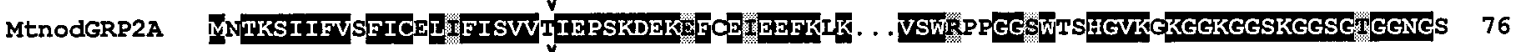

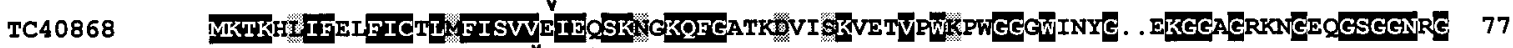
TC35313 MKIK. MFMFLYFCALIIISVVAIEISKNEKCSGEAKESFINIEVDGKVRWSEGRATWRSGSGNQNEEHNSIINYSDNEK 78

TC41363 GEEPËEGSRGGËNGKFGEGEGWENEGGGGGEGGGGEEEEGFSEYEETGEYP

MTN29 GGKRREA. . RRGRNRGNWGGGNEYSSVREGYEGPGGGYEGRGGEEGGEAGVHEENFERGENEEPGLG 139

MSNOdGRP2B EGGAQGGGGQIDGGXDKGSGLDARE. . GENNKIGWGYLKN

MtnOdGRP2B EGGAQGGGQIIGGGDKGSGLDARGGGRGENNNIGWGYLKN 117

$\begin{array}{lr}\text { MSNOdGRP2A EGGAQGGGQIEGGKDKSGLDXRE } & 101\end{array}$

$\begin{array}{lr}\text { MtnodGRP2A EGGAQGGEQITGGNDKESELDGGGGGGDIIK } & 109\end{array}$

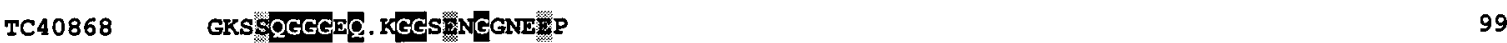

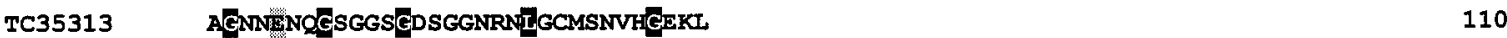

\section{C}

MtnOdGRP1A MtnOdGRP1B MtnodGRP1C MsnodGRP1 33

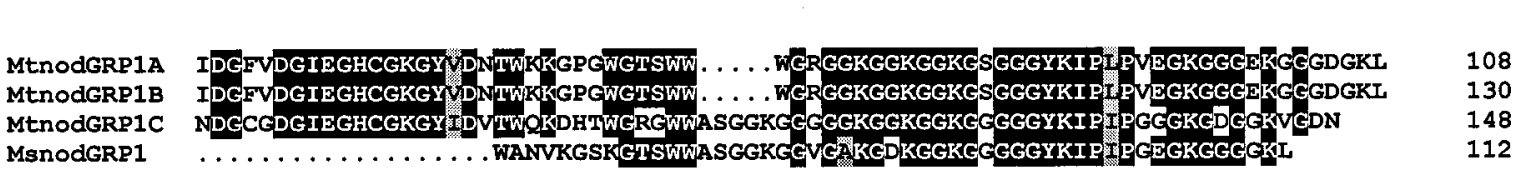

Fig. 2. Multiple alignments of homologous glycine-rich proteins. A, Comparison of the MsnodGRP3, Vfenod-GRP5 (VfGRP5), and alfalfa NMs22 proteins. Repetitive blocks of MsnodGRP3 are marked with horizontal arrows; conservative amino acid motifs are between vertical arrows. B, Multiple alignments of MsnodGRP2A, MsnodGRP2B, MtnodGRP2A, MtnodGRP2B, MtN29, and proteins deduced from the Medicago truncatula TC40868, TC41363, and TC35313 expressed sequence tags. The end of putative signal peptides is marked with arrows in each sequence. C, Comparison of MsnodGRP1 and MtnodGRP1s. Identical amino acid residues are on black background, while similar amino acids are shown on gray. In $\mathbf{B}$, and $\mathbf{C}$, amino acid residues conserved in at least four sequences are indicated. 
conservation of the WRDWGG oligopeptide, while VfenodGRP5 had only two repeats but with the same WRDWGGSFW oligopeptide sequence as MsnodGRP3. In contrast to MsnodGRP3, MsnodGRP1, lacking the characteristic glycinerich repeats, shared no homology with known proteins.

MsnodGRP2A and MsnodGRP2B showed only a weak homology to Vfenod-GRP4 (62\% similarity and 43\% identity with MsnodGRP2A, 64\% similarity and 46\% identity with MsnodGRP2B). The MsnodGRP2A and MsnodGRP2B proteins exhibited $85 \%$ similarity and $80 \%$ identity to each other (Fig. 2B). To determine whether MsnodGRP2A and $M s n o d G R P 2 B$ represented allelic variants in the tetraploid, allogamous $M$. sativa genome or two distinct genes, a $M$. truncatula nodule cDNA library was screened with the $M$. sativa cDNA clones. This resulted in the isolation of both MtnodGRP2A and MtnodGRP2B (accession numbers AF498993 and AF498994, respectively) cDNA clones, revealing that these GRPs are encoded by different genes. The signal peptides of MtnodGRP2A and MtnodGRP2B proteins shared significant homology with the putative signal peptides of MtN29 (Gamas et al. 1996) and a few other GRP predicted from EST sequences (TC40868, TC41363, and TC35313) present in the M. truncatula databases (Fig. 2B). The respective genes also encoded small proteins that exhibited significantly high glycine content beside the putative hydrophobic signal peptide. The homology values between the signal peptides of these GRPs were around $80 \%$ or higher, while their glycine-rich domains displayed only 60 to $65 \%$ similarity and 30 to $35 \%$ identity. By screening the $M$. truncatula nodule cDNA library with the MsnodGRP1 probe, we obtained three variants, MtnodGRPIA, MtnodGRPIB, and MtnodGRP1C (accession numbers AF498990, AF498991, and AF498992, respectively). The encoded proteins were homologous to MsnodGRP1, both at the N-terminal signal peptide region and in the $\mathrm{C}$-terminal part. Downstream of the signal peptide, one oligopeptide block was conserved in MtnodGRP1B, MtnodGRP1C, and MsnodGRP1, but not in MtnodGRP1A; while in the central region a 30-amino-acidlong sequence was present in the MtnodGRPs but absent in MsnodGRP1 (Fig. 2C).

Expression of the four MsnodGRPs is nodule specific, requires rhizobial infection, and is unaffected by other GRP inducers.

Expression of the isolated GRP genes was investigated by Northern analysis using total RNA isolated from different organs of alfalfa (flower, root, hypocotyl, leaf, and stem) including young (7-day-old) and mature (21-day-old) nodules, as well as spontaneous nodules developed in the absence of $S$. meliloti. RNA samples $(5 \mu \mathrm{g})$ were used for Northern hybridization, and equal loading was visualized by ethidium-bromide staining. The blots were also hybridized with the constitutive Msc27 gene probe, which can be reliably used for normalization of the signals in roots and nodules in contrast to aerial plant organs, which express $M s c 27$ at different levels. No expression of any of the four GRP genes was detected in flowers, leaves, stems, hypocotyls, roots, or spontaneous nodules. In contrast, all were expressed in $S$. meliloti-induced nodules. Expression of the MsnodGRP1 as well as the MsnodGRP2A and MsnodGRP2B genes was predominant in young nodules, while the MsnodGRP3 transcript accumulation increased during nodule development (Fig. 3A). The MsnodGRPl probe revealed two transcripts, the smaller one corresponded to the size of the MsnodGRP1 cDNA. The larger one might originate from a related gene; however, screening of the $M$. sativa subsp. varia A2 young nodule cDNA library resulted only in the isolation of MsnodGRP1 in spite of the similar abundance of the two tran- scripts. Therefore, it may be that both transcripts derive from the MsnodGRP1 gene but that they differ in their polyadenylation or alternative splicing during RNA maturation.

Northern analysis of GRP genes thus revealed that they were real nodulin genes. The lack of expression in spontaneous nodules demonstrated that bacterial infection is indispensable for the induction of MsnodGRP1, MsnodGRP2A, MsnodGRP2B, and MsnodGRP3 genes. The possible requirement of bacteria for the expression of MsnodGRP genes was further studied with the help of two $S$. meliloti mutants that were wild type in respect to Nod factor production and initiation of nodule organogenesis but were defective in the invasion process or in their differentiation to nitrogen-fixing bacteroids. AK1492 is a dou-

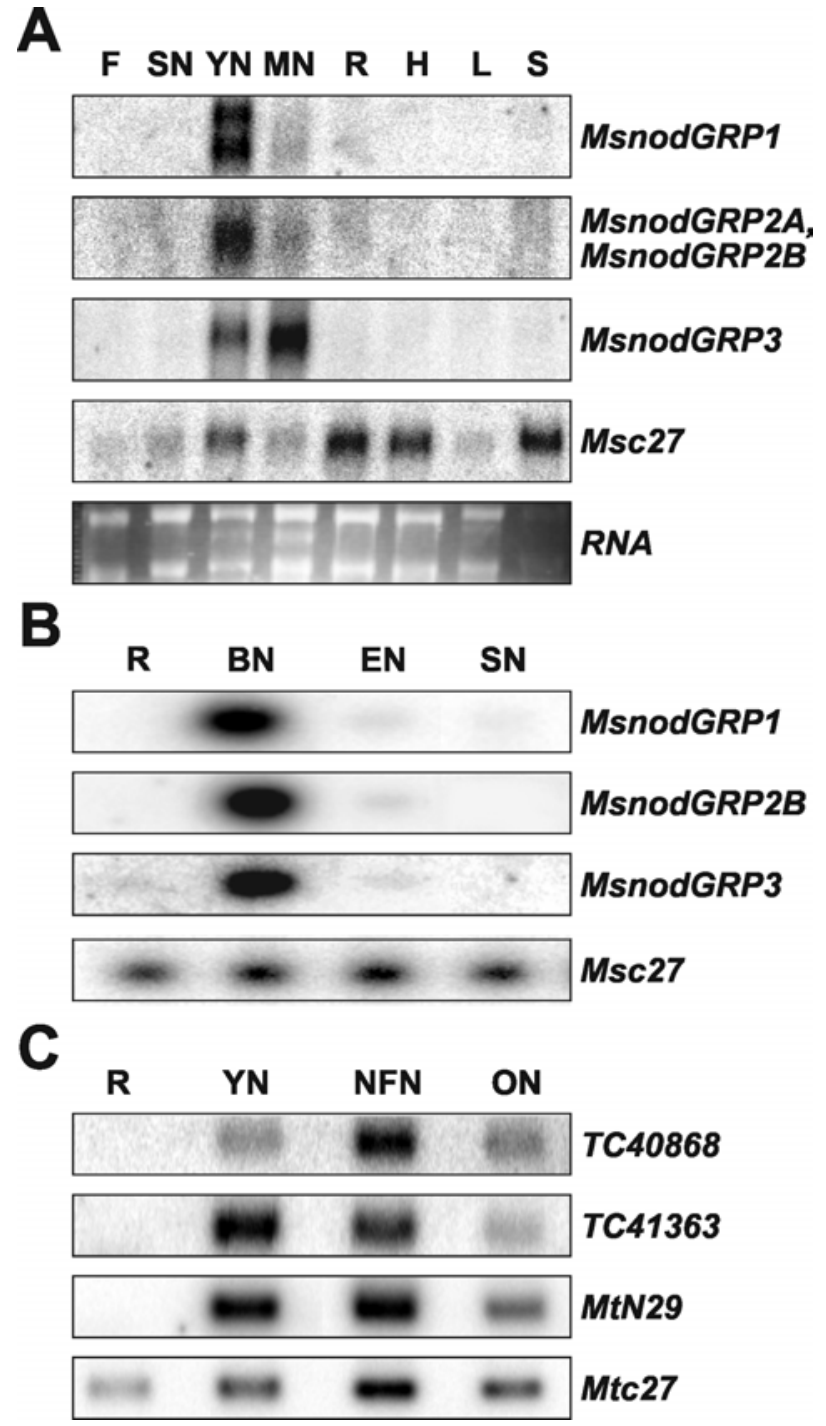

Fig. 3. Expression of Ms/MtnodGRP genes is nodule specific and requires Sinorhizobium meliloti infection. Hybridization probes are indicated on the right. Msc/Mtc27 and ethidium bromide stain of RNA gel served as controls for loading. A, Northern analysis of nodGRPs in alfalfa flowers $(\mathrm{F})$, spontaneous nodules $(\mathrm{SN})$, young nodules at $7 \mathrm{dpi}$ $(\mathrm{YN})$, mature nodules at $21 \mathrm{dpi}(\mathrm{MN})$, roots $(\mathrm{R})$, hypocotyls $(\mathrm{H})$, leaves (L), and stems (S). B, Reverse transcription-polymerase chain reaction (RT-PCR) analysis of MsnodGRP expression in alfalfa roots (R), nodules induced by $\mathrm{Bac}^{-}(\mathrm{BN})$ and $\mathrm{Exo}^{-}(\mathrm{EN}) S$. meliloti mutants and by spontaneous nodules $(\mathrm{SN})$ developed in the absence of rhizobia. C, RT-PCR results of the expression of TC40868, TC41363, and MtN29 genes in Medicago truncatula $\mathrm{R}-108$ roots $(\mathrm{R})$, young nodules collected 9 dpi (YN), nitrogen-fixing nodules collected $20 \mathrm{dpi}$ (NFN), and nodules at 29 dpi (ON). 

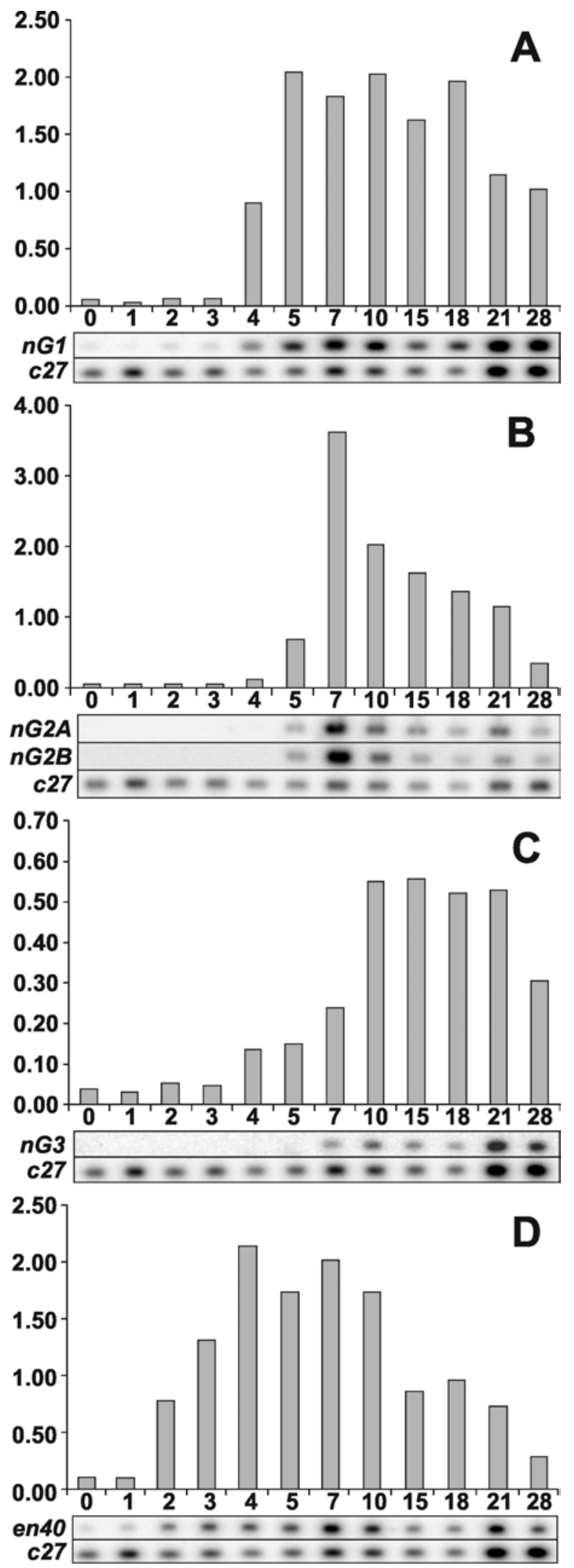

Fig. 4. Reverse transcription-polymerase chain reaction (RT-PCR) analysis of MsnodGRP and Msenod40 genes in the course of nodule development. cDNAs were synthesized from total RNAs isolated from roots and nodules at $0,1,2,3,4,5,7,10,15,18,21$, and $28 \mathrm{dpi}$ and were used for PCR with gene-specific primers A, $n G 1$ for MsnodGRP1, B, $n G 2 A$ and $B$ for MsnodGRP2A and B, respectively, $\mathbf{C}, n G 3$ for MsnodGRP3, and $\mathbf{D}$, en40 for Msenod40. PCR products were detected by Southern hybridization. Histograms show the normalized hybridization signal intensities calculated on the basis of $M s c 27$ (c27) signals. ble mutant of $S$. meliloti 41, unable to produce wild-type exopolysaccharide (EPS) and capsular polysaccharide (KPS) and thereby impaired in the infection process (Exo- $\mathrm{Kps}^{-} \mathrm{Inf}$ mutant). The other mutant, strain 8368, produced wild-type polysaccharides but was defective in bacteroid differentiation $\left(\mathrm{Bac}^{-}\right)$. In the nodules induced by strain AK1492, the expression of all four GRPs was drastically reduced, in contrast to the nodules induced by the $\mathrm{Bac}^{-}$mutant in which expression of $G R P$ genes was unaffected (Fig. 3B). These results indicated that the expression of these GRP genes might be controlled by bacterial polysaccharide signals or that they might require invasion of the nodule cell by Rhizobium, or both.

Since it was shown that expression levels of several GRP genes were regulated by hormones and osmotic stress, we investigated whether expression of these nodule-specific, rhizobia-induced, GRP genes was induced by factors other than rhizobia. Treatment of alfalfa roots with naphthalene acetic acid (NAA), absicic acid (ABA), kinetin and gibberelic acid $\left(\mathrm{GA}_{3}\right)$, or with exposure to heat $\left(37^{\circ} \mathrm{C}\right)$ and cold $\left(4^{\circ} \mathrm{C}\right)$ shocks, hypoxia $\left(+\mathrm{H}_{2} \mathrm{O}\right)$, and drought $\left(-\mathrm{H}_{2} \mathrm{O}\right)$ stresses, however, did not result in the expression of the nodule-specific GRPs tested by reverse transcription-polymerase chain reaction (RT-PCR) (data not shown). These results further strengthen the nodulespecific function of these GRPs.

\section{MsnodGRP2A- and MsnodGRP2B-related M. truncatula genes are also nodule specific.}

Although the proteins encoded by the M. truncatula ESTs TC40868 and TC41363 as well as MtN29 exhibited homology to Ms/MtnodGRP2 only in the signal peptide region, their relatively high glycine content prompted us to study their expression profiles. Using specific oligos, each gene was amplified from cDNAs prepared from mRNAs isolated from $M$. truncatula R108 nodules of different ages and roots treated with Nod factors (Fig. 3C). No expression of any of these genes was detected in roots, while all of them were induced during nodule development, albeit with different kinetics. The maximal transcript level for TC40868 was detected in the nitrogen-fixing nodules, while for TC41363, it was in the young nodules. Expression of MtN29 was similar in the young and the nitrogenfixing nodules. As nodules aged, the transcript level of all the three genes declined.

\section{MsnodGRP nodulins exhibit different temporal expression patterns.}

The induction of GRP genes during nodule development was studied by RT-PCR experiments. Total RNA samples for cDNA preparation were isolated from roots of sterile seedlings grown on agar plates in nitrogen-free medium after inoculation of roots with $S$. meliloti at 0 to 3 days post inoculation (dpi), from nodule primordia (4 to $5 \mathrm{dpi}$ ), and from young ( 7 to 10 dpi) and mature nodules (15 to $28 \mathrm{dpi}$ ). Accumulation of the $G R P$ mRNAs was measured during nodule development and compared to that of $M s c 27$ used as a control for the quantification of the cDNAs in the different samples (Fig. 4). As a control for nodule development, expression of early nodulin gene Msenod40 was included (Fig. 4D). In the case of MsnodGRP1, a weak background signal was detectable in the root, while a significant increase in mRNA abundance was observed at 4 dpi, reaching its maximum at $5 \mathrm{dpi}$, which was maintained during the development and maturation of nodules (Fig. 4A). MsnodGRP $2 A$ and MsnodGRP $2 B$ had no background signal in the root and were strongly induced at 5 dpi (1 day later than MsnodGRP1), with a maximal transcript accumulation at $7 \mathrm{dpi}$ that then decreased gradually (Fig. 4B). Induction of the MsnodGRP3 gene started at day 7 and was maximal through the active, nitrogen-fixing period (Fig. 4C). In comparison with 
nodGRP genes, expression of Msenod40 was induced at $2 \mathrm{dpi}$, thus 2 days earlier than MsnodGRP1, but both of them before nitrogen fixation. Expression of MsnodGRP2A and MsnodGRP2B exhibited a three-day delay and MsnodGRP3 a five-day delay compared with Msenod40. These differences in the expression kinetics indicated that MsnodGRPs might be involved in consecutive stages of nodule development with different functions.

\section{Distinct spatial expression patterns} of the GRP nodulin genes.

To determine the spatial expression patterns of GRP genes in nodules, in situ hybridizations were carried out with sense and antisense RNA probes that were synthesized from cDNAs encoding the glycine-rich domains and that were specific for MsnodGRP1, MsnodGRP2, and MsnodGRP3 but did not distinguish MsnodGRP2A from MsnodGRP2B. The sections
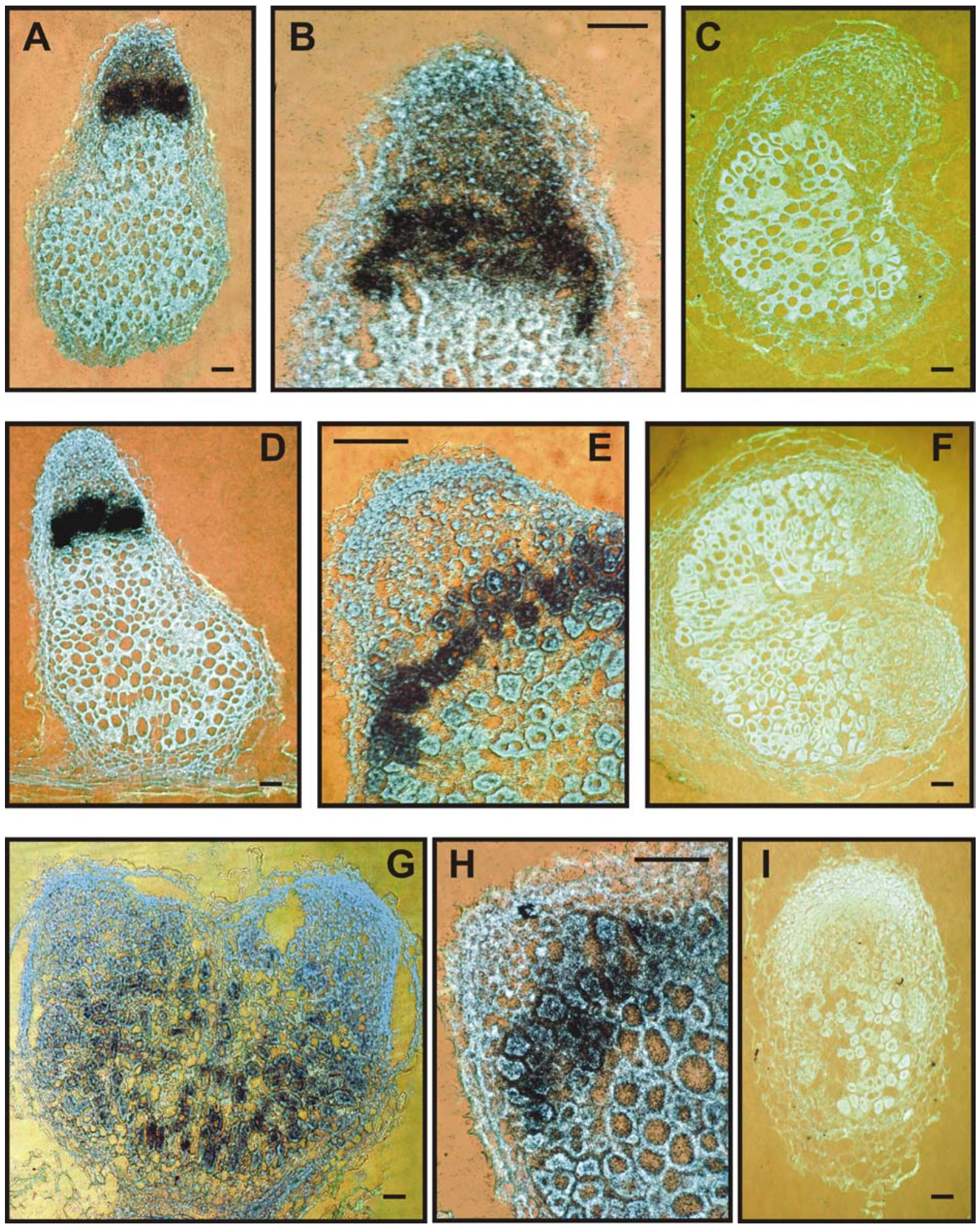

Fig. 5. Localization of MsnodGRP transcripts in Medicago sativa nodules by in situ hybridization. Hybridization of antisense riboprobes specific for A and $\mathbf{B}$, MsnodGRP1, D and E, MsnodGRP2, and $\mathbf{G}$ and $\mathbf{H}$, MsnodGRP3. Hybridization with sense probes of $\mathbf{C}$, MsnodGRP1, F, MsnodGRP2, and I, MsnodGRP3. Bars correspond to $200 \mu \mathrm{m}$. 
derived from alfalfa nodules of different ages that represented all stages of nodule development. The MsnodGRPl transcripts were localized in the nodule apex in zone II, while no signals were detected in the nitrogen fixation zone III (Fig. 5A). In zone II, the highest expression level was found in cells adjacent to interzone II-III, where MsnodGRPI transcripts were also present (Fig. 5B). The MsnodGRP2 gene exhibited expression exclusively in interzone II-III (Fig. 5D) and predominantly in a single cell layer (Fig. 5E). In the case of MsnodGRP3, no hybridization signals were detected in the nodule apex, and the expression appeared to be associated with the nitrogen-fixing symbiotic cells in zone III (Fig. 5G). In a few cases, the hybridization signals were stronger in the early symbiotic zone than in the older cells of zone III (Fig. 5H). The lack of background signals on nodule sections hybridized with the sense RNA probes (Fig. 5C, 5F, 5I) and the restricted signal localizations obtained with the antisense probes confirmed that these hybridizations were specific for all

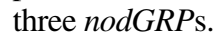

\section{DISCUSSION}

In this paper, we presented the isolation and characterization of cDNAs coding for different GRP from $M$. sativa and $M$. truncatula nodules. These proteins, like most GRP, were composed of a secretory signal peptide followed by a glycine-rich domain. However, their glycine content was lower (20 to 30\%) than that of other GRPs (where it was around $80 \%$ or even higher) although still significantly higher than in other proteins not considered rich in glycine. In addition, these nodulin genes were exclusively expressed in Medicago nodules, confirming that they represent a novel subfamily of the GRP genes.

All nodule-specific GRPs described in this study were small proteins, and most of them lacked the glycine-rich repetitive motifs characteristic for non-nodule-specific GRPs (SachettoMartins et al. 2000). In these proteins, only few repetitions of a GGX sequence were present. MsnodGRP3 was the only one that contained four repeats of an oligopeptide with the WRDWGGSFW motif. This motif was present in two other nodule-specific proteins, NMs22 (Ganter et al. 1998) and Vfenod-GRP5 (Schröder et al. 1997) that differed from MsnodGRP3, however, in the number of repeats. The protein encoded by a $M$. truncatula EST, TC40954, and predicted as a homologue of NMs22 (NMt22) had only two oligopeptides of the four repeats, likewise Vfenod-GRP5. These differences suggest that the functions of these nodulins are distinct from that of MsnodGRP3. Moreover, database search carried out with the signal peptide of MsnodGRP3 revealed similarity to the signal peptide of nodulin DD17, a recently available sequence in the database (accession number CAC84521) from another leguminous plant, Trifolium repens, which also contained several glycine residues at carboxy terminal region.

The primary structures of MsnodGRP2A and MsnodGRP2B were highly homologous and were encoded by different genes, as it was revealed by isolation of the corresponding $M$. truncatula clones. These proteins showed a weak homology to Vfnod-GRP4, excluding the signal peptide that was present in alfalfa but not in broad bean. In contrast, the signal peptides of MsnodGRP2A and MsnodGRP2B exhibited significant homologies with three proteins predicted from $M$. truncatula EST sequences (TC40868, TC41363, and TC35313). These proteins also displayed glycine-rich domains but without homology to Ms/MtnodGRP2A and Ms/MtnodGRP2B or to each other. One of the signal peptide homologues was MtN29, identified as a nodulin (Gamas et al. 1996). Moreover, a similar signal peptide was present in the early nodulin Enod7 (accession number CAA63660) from Pisum sativum, but the glycine content of this putative protein was relatively low.
MsnodGRP1 was the only GRP that did not show homology to other proteins in the databases. Screening of a $M$. truncatula library with the MsnodGRP1 probe resulted in three homologues but not the real orthologues of the alfalfa protein. The homology was extended to the signal peptide and the carboxy terminal part of the proteins, while the central regions were less conserved (Fig. 2C).

Relatedness of the GRP nodulins is presented in a phylogenetic tree (Fig. 6). As it was expected, MsnodGRP1 and the MsnodGRP1-related $M$. truncatula proteins MtnodGRP1A, MtnodGRP1B, and MtnodGRP1C formed one group. Interestingly, the signal peptide homologues of Ms/MtnodGRP2s, TC40868, TC41363, and TC35313, were on distinct branches from the Ms/MtnodGRP2 proteins. TC40868 and TC41363 proteins were in the group of Ms/MtnodGRP3 proteins. This group also included NMs22/NMt22, Vfenod-GRP5, DD17, and Vfenod-GRP3. TC35313 was more related to nodGRP1 proteins. The MsnodGRP2 and MtnodGRP2 proteins were related to Vfnod-GRP4. MtN29, the signal peptide homologue of Ms/MtnodGRP2s, exhibited distinct origin. Taking into account that most of these nodGRPs showed no homology to other GRP, these nodulation-related GRPs represent both a novel class of GRPs and a novel group of nodulins.

Expression of all Medicago nodGRPs was nodule specific and required infection of rhizobia; however, their activation and maximal transcript accumulation differed during nodulation. The MsnodGRPl gene was induced at $4 \mathrm{dpi}$, two days later than Msenod40, an early nodulin gene that is required for cell proliferation and for initiation of the nodule primordium (Crespi et al. 1994). At day 4, nodule primordium differentiates to a meristem and an invasion zone in which infection of plant cells with bacteria occurs. Consistently with the expression kinetics, MsnodGRPl transcripts were localized by in situ hybridization along all cell layers of zone II, where they were particularly abundant in distal cell layers and in interzone II-III but were absent in zone III. This expression profile suggests that nodGRP1 might be involved in differentiation of symbiotic tissue. This localized zone II-associated expression pattern also explains the decrease in the transcript level during nodule maturation detected by Northern analysis and RT-PCR. By nodule growth, the mass of zone III increases while that of zone II remains constant, thus, although the expression level of MsnodGRP1 does not change, the concentration of their transcripts decreases with the increasing amount of mRNAs from zone III. From M. truncatula, we have isolated three homologous nodGRP1 cDNAs (MtnodGRPIA, MtnodGRP1B, and MtnodGRP1C). This suggests that additional nodGRP1-related proteins might be produced in $M$. sativa nodules as well.

Transcripts of MsnodGRP2A and MsnodGRP2B were indistinguishable by Northern analysis, due to the cross-hybridization of the genes. Using specific oligos for each gene, RT-PCR experiments demonstrated that neither of the two genes expressed in roots and that both were activated at 5 dpi. At days 5 to 7, the distal cell layers of zone II are converted into nitrogen-fixing symbiotic cells. During this period, transcript accumulation of MsnodGRP2s increased fivefold and then decreased gradually. In situ hybridization revealed the exclusive presence of MsnodGRP2 transcripts in interzone II-III in two or a maximum of three cell layers, where the most drastic and probably the most critical developmental changes occur prior to terminal differentiation of nitrogen-fixing symbiotic cells. This expression profile is in perfect agreement with transient expression of MsnodGRP2 detected by RT-PCR analysis, revealing an illusive and more drastic decrease in MsnodGRP2 transcript levels than in those of MsnodGRPl by nodule growth and maturation. As revealed by RT-PCR analysis of root and nodule RNAs, expression of the signal peptide homo- 
logues of Ms/MtnodGRP2s (TC40868, TC41363, and MtN29) was nodule specific as well.

Northern analysis demonstrated that expression of MsnodGRP3 was induced at $7 \mathrm{dpi}$ in the young nitrogen-fixing nodules and was predominant during the period of active nitrogen fixation. By in situ hybridization, the MsnodGRP3 transcripts were detected in the nitrogen-fixing cells in zone III that correlated with the relatively retarded induction of MsnodGRP3 compared with MsnodGRP1 and MsnodGRP $2 A / B$ and with the elevated transcript levels through the active nitrogen-fixing period.

The expression pattern of nodGRP genes in nodules induced by $S$. meliloti mutant strains demonstrated that activation of all the nodGRP genes we studied required bacterial infection but was indifferent of bacteroid differentiation. Differential activation kinetics of these genes and distinct spatial localization of the nodGRP mRNAs indicate that they might have distinct functions during nodule organogenesis. Their expression in particular zones and cell layers suggests that these nodGRPs may be involved in signaling processes, similar to AtGRP3, which is a binding partner of the cell wall-integrated WAK1 protein that is responsible for development of leaf cells (Kohorn 2001). During nodule differentiation, when bacteria invade the host cells, nodGRP1 and nodGRP2A/B might function or be involved as signals for the production of specific molecular structures (such as peribacteriod membranes) that are required for the development of symbiotic cells. The function of nodGRP2A/B appears to be, however, more specific and might be required for terminal differentiation of symbiotic cells. In contrast to nodGRP1 and nodGRP2A/B, nodGRP3 plays a role in a later stage of symbiosis associated with nitrogen fixation. The possible involvement of these nodGRPs in Rhizobium-elicited signaling processes is in line with their exclusive nodule-specific expression and with the finding that none of the MsnodGRPs were induced by biotic and abiotic regulators of the other, non-nodule-specific GRPs.

Elucidation of the real nodGRP functions would necessitate the analysis of knock out $M$. truncatula mutants. Although, construction of tagged $M$. truncatula mutant banks is in progress in the laboratory, their creation will take several years. Therefore, as alternative approaches for gene silencing, we have started the generation of antisense and RNAi transgenic $M$. truncatula plants to evaluate the importance of nodGRPs in nodule development. For transgenic studies in nodulation, isolation of the promoter regions of nodGRP genes could also be extremely useful to drive localized and nodule-specific gene expression. The identification of interacting protein partners of the nodGRPs is expected to give an insight into putative signaling pathways. Therefore, yeast two-hybrid screens will be performed for isolation of nodGRP partners. All these future studies will contribute to elucidation of the biological functions of nodGRPs, which appear to be specific for leguminous plants forming indeterminate nodules.

\section{MATERIALS AND METHODS}

\section{Bacterial strains.}

In the $M$. sativa and $M$. truncatula nodulation assays, the following Rhizobium strains were used: wild-type $S$. meliloti $\mathrm{Rm} 41$; Sm8368, a $\mathrm{Bac}^{-}$derivate of Sm1021 carrying a TnphoA insertion in the bacA gene (Glazebrook et al. 1993); and AK1492, an $\mathrm{Eps}^{-} \mathrm{Kps}^{-} \mathrm{Fix}^{-}$mutant strain of Rm41 containing a deletion in $E x o B$ and a Tn5 insertion in the fix23 region encoding capsular polysaccharide production (Kondorosi et al. 1984; Putnoky et al. 1988). Bacteria were grown at $30^{\circ} \mathrm{C}$ in tryptone-agar medium (Orosz et al. 1973) that was supplemented for the mutant strains with kanamycin $\left(100 \mu \mathrm{g} \mathrm{ml}^{-1}\right)$.
Plant material.

Seeds of alfalfa (M. sativa subsp. sativa cv. Sitel) and M. truncatula ecotype R-108 (Hoffman et al. 1997) were sterilized by gentle agitation in 20\% Pasti-chlor (Argos, Bondoufle, France) for $30 \mathrm{~min}$ at room temperature, then washed four times with sterile water, and germinated in the dark on $1 \%$ water agar plates for $24 \mathrm{~h}$. For the time course experiments, the seedlings were transferred to square petri dishes containing nitrogen-free solution (Crespi et al. 1994) with $1 \%$ agar and incubated in a growth chamber at $28^{\circ} \mathrm{C}$. After 4 to 5 days, the roots were spot-inoculated with $S$. meliloti $\mathrm{Rm} 41$ and samples were collected from 1-, 2-, 3-, 4-, 5-, 7-, 10-, 15-, 18-, 21-, and 28-day-old nodule primordia or nodules, as well as from noninoculated roots as a control.

For large scale RNA isolation, seedlings were grown in aeroponic factories (Hoffman et al. 1997). For nodulation assay, seedlings were grown for one week in nitrogen-limiting medium and then inoculated with wild-type or mutant S. meliloti strains. Wild-type nodules were harvested at 7 and 21 dpi from $M$. sativa and at 9, 20, and 29 dpi from M. truncatula. In the case of $\mathrm{Bac}^{-}$and $\mathrm{Exo}^{-}$mutants, the nodules were collected 2 to 3 weeks after infection. Flowers, roots, hypocotyls, leaves, and stems of alfalfa were collected from 2- to 3-week-old plants grown in nitrogen-containing solution. Spontaneous nodules were collected from the highly embryogenic $M$. sativa subsp. varia A2 line after three weeks of nitrogen starvation.

\section{Library screening and sequencing.}

Alfalfa GRP cDNA clones were obtained by screening a cDNA library of young $M$. sativa subsp. varia A2 nodules (Crespi et al., 1994) using the Vfnod-GRP1, Vfenod-GRP2, Vfenod-GRP3, Vfnod-GRP4, and Vfenod-GRP5 cDNA probes of Vicia faba (Küster et al. 1995; Schröder et al. 1997). MtnodGRP2A and MtnodGRP2B cDNA clones were isolated from a $M$. truncatula R-108 nodule cDNA library (Györgyey et al. 2000). The cDNA clones were sequenced by the chain terminator method (Pharmacia kit; Pharmacia SAS, Saint Quentin-en-Yveline, France) using an automatic laser sequencer 373A (Applied Biosystems, Foster City, CA, U.S.A.).

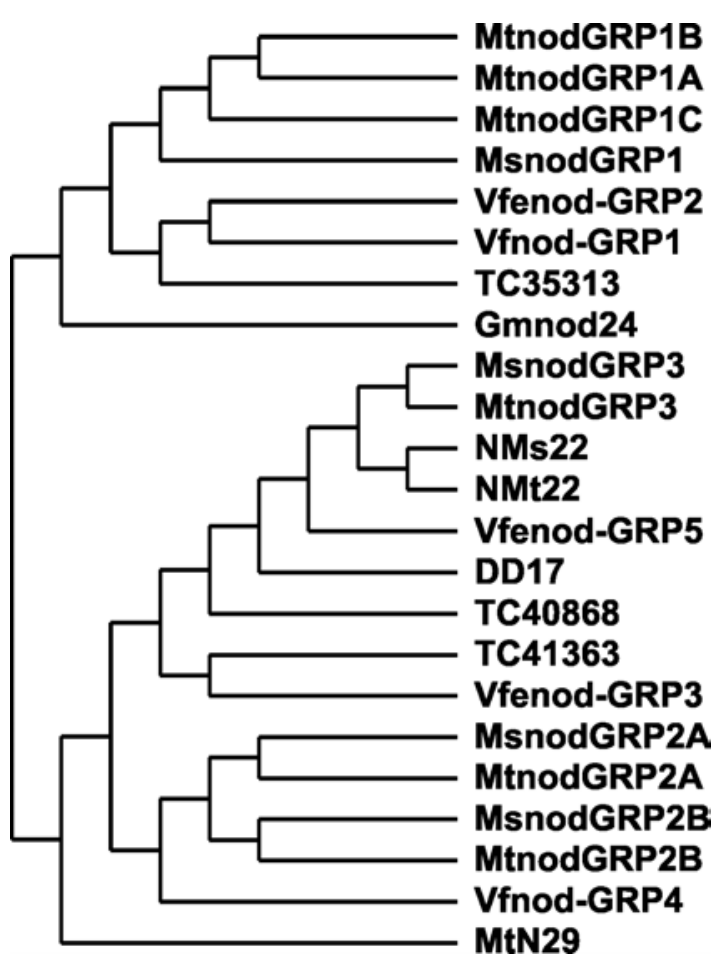

Fig. 6. Phylogenetical tree of MsnodGRPs and their homologues. 


\section{Northern and RT-PCR analysis}

Total RNA was isolated from alfalfa by the RNeasy plant mini kit (Qiagen, Hilden, Germany) from nodules, roots, leaves, stems, flowers, and hypocotyls. For Northern blots, 8 $\mu \mathrm{g}$ of total RNA was used. For RT-PCR, cDNAs were synthesized by the treatment of $1 \mu \mathrm{g}$ of total RNA with DNase (FPLC pure; Amersham Pharmacia Biotech, Uppsala, Sweden) that was retrotranscribed in the presence of M-MLV reverse transcriptase (Promega, Madison, WI, U.S.A.), RNase inhibitor (RNasin, Promega) and oligo-dT primers. Msc27 (Györgyey et al. 1991) and $M t c 27$ were amplified in 17 cycles $\left(94^{\circ} \mathrm{C}\right.$ for 30 s, $55^{\circ} \mathrm{C}$ for $30 \mathrm{~s}$, and $72^{\circ} \mathrm{C}$ for $1 \mathrm{~min}$ ) while MsnodGRP1, MsnodGRP2A, MsnodGRP2B, MsnodGRP3, TC40868, $T C 41363$, and $M t N 29$ were amplified in 21 cycles. After agarose gel electrophoresis, RT-PCR products were blotted on nylon membranes (BioTrans; ICN, Costa Mesa, CA, U.S.A.). $\alpha\left[{ }^{32} \mathrm{P}\right]-\mathrm{dCTP}$ radiolabeled DNA probes were generated by random priming (Amersham Pharmacia Biotech). Hybridizations were performed in $0.5 \mathrm{M}$ Na-phosphate, $\mathrm{pH} 7.2$, and $7 \%$ sodium dodecyl sulfate (SDS) buffer (Church and Gilbert 1984) for $36 \mathrm{~h}$ at $65^{\circ} \mathrm{C}$; then the membranes were washed twice with $2 \times \mathrm{SSC}(1 \times \mathrm{SSC}$ is $0.15 \mathrm{M} \mathrm{NaCl}$ plus $0.015 \mathrm{M}$ sodium citrate) and $0.1 \%$ SDS and with $1 \times$ SSC and $0.1 \%$ SDS. Hybridization signals were exposed on phosphor-imager screens. For hybridization signals, intensities were quantified and normalized by Phosphor-imager STORM (Molecular Dynamics, Sunnyvale, CA, U.S.A.) and specific computer packages.

\section{In situ hybridization.}

In situ hybridizations were carried out according to de Almeida Engler and associates (2001) using 200-bp-long ${ }^{35} \mathrm{~S}$ labeled sense and antisense riboprobes (corresponding to the glycine-rich domains). Slides were exposed to Kodak NTB-2 emulsion, and signals were detected with fluorescent-light microscope and CDD-camera.

\section{ACKNOWLEDGMENTS}

We are grateful to P. Mergaert and K. Kelemen for providing cDNAs for nodulation kinetics and analysis of stress and hormone effects. We thank M. Redondo-Nieto for the preparation of the figures in the manuscript. Z. K. was supported by a co-tutelle Ph.D. grant of the French (C.R.O.U.S.) and Hungarian governments and by the "Jumelage" program between the C.N.R.S. and the Hungarian Academy of Sciences, while J.M.V. was supported by the TMR-Marie Curie fellowship program.

\section{LITERATURE CITED}

Anderson, C. M., Wagner, T. A., Perret, M., He, Z., He, D., and Kohorn, B. D. 2001. WAKs: Cell wall-associated kinases linking the cytoplasm to the extracellular matrix. Plant Mol. Biol. 47:197-206.

Carpenter, C. D., Kreps, J. A., and Simon, A. E. 1994. Genes encoding glycine-rich Arabidopsis thaliana proteins with RNA-binding motifs are influenced by cold treatment and an endogenous circadian rhythm. Plant Physiol. 104:1015-1025.

Cassab, G. I. 1998. Plant cell wall proteins. Annu. Rev. Plant Physiol. Plant Mol. Biol. 49:281-309.

Cheon, C. I., Hong, Z., and Verma, D. P. 1994. Nodulin-24 follows a novel pathway for integration into the peribacteroid membrane in soybean root nodules. J. Biol. Chem. 269:6598-6602.

Church, G. M. and Gilbert, W. 1984. Genomic sequencing. Proc. Natl. Acad. Sci. U.S.A. 81:1991-1995.

Condit, C. M. 1993. Developmental expression and localization of petunia glycine-rich protein 1. Plant Cell 5:277-288.

Crespi, M. and Gálvez, S. 2000. Molecular Mechanisms in Root Nodule Development. J. Plant Growth Regul. 19:155-166.

Crespi, M. D., Jurkevitch, E., Poiret, M., d'Aubenton Carafa, Y., Petrovics, G., Kondorosi, E., and Kondorosi, A. 1994. Enod40, a gene expressed during nodule organogenesis, codes for a non-translatable RNA involved in plant growth. EMBO (Eur. Mol. Biol. Organ.) J.
13:5099-5112.

Cretin, C., and Puigdomenech, P. 1990. Glycine-rich RNA-binding proteins from Sorghum vulgare. Plant Mol. Biol. 15:783-785.

de Almeida Engler, J., De Groodt, R., Van Montagu, M., Engler, G. 2001. In situ hybridization to mRNA of Arabidopsis tissue sections. Methods 23:325-334.

de Oliveira, D. E., Seurinck, J., Inze, D., Van Montagu, M., and Botterman, J. 1990. Differential expression of five Arabidopsis genes encoding glycine-rich proteins. Plant Cell 2:427-436.

Dreyfuss, G., Swanson, M. S., and Pinol-Roma, S. 1988. Heterogeneous nuclear ribonucleoprotein particles and the pathway of mRNA formation. Trends Biochem. Sci. 13:86-91.

Gamas, P., de Carvalho Niebel, F., Lescure, N., and Cullimore, J. 1996. Use of a subtractive hybridization approach to identify new Medicago truncatula genes induced during root nodule development. Mol. PlantMicrobe Interact. 9:233-242.

Ganter, G., Raja, S., and Dunn, K. 1998. The cDNA sequence of Nms-22 (accession no. AF030252), a gene whose message is specific to the infected cells of alfalfa (Medicago sativa) root nodules. Plant Physiol. 116:446.

Glazebrook, J., Ichige, A., and Walker, G. C. 1993. A Rhizobium meliloti homolog of the Escherichia coli peptide-antibiotic transport protein SbmA is essential for bacteroid development. Genes Dev. 7:14851497.

Gómez, J., Sánchez-Martínez, D., Stiefel, V., Rigau, J., Puigdomenech, P., and Pages, M. 1988. A gene induced by the plant hormone abscisic acid in response to water stress encodes a glycine-rich protein. Nature 334:262-264.

Györgyey, J., Gartner, A., Németh, K., Magyar, Z., Hirt, H., HeberleBors, E., and Dudits, D. 1991. Alfalfa heat shock genes are differentially expressed during somatic embryogenesis. Plant Mol. Biol. 16:999-1007.

Györgyey, J., Vaubert, D., Jiménez-Zurdo, J. I., Charon, C., Troussard, L., Kondorosi, A., and Kondorosi, E. 2000. Analysis of Medicago truncatula nodule expressed sequence tags. Mol. Plant-Microbe Interact. 13:62-71.

Hammond-Kosack, K. E., and Jones, J. D. G. 1996. Resistance gene-dependent plant defense responses. Plant Cell 8:1773-1791.

Harrak, H., Chamberland, H., Plante, M., Bellemare, G., Lafontaine, J. G., and Tabaeizadeh, Z. 1999. A proline-, threonine-, and glycine-rich protein down-regulated by drought is localized in the cell wall of $x y-$ lem elements. Plant Physiol. 121:557-564.

Heintzen, C., Melzer, S., Fischer, R., Kappeler, S., Apel, K., and Staiger, D. 1994. A light- and temperature-entrained circadian clock controls expression of transcripts encoding nuclear proteins with homology to RNA-binding proteins in meristematic tissue. Plant J. 5:799-813.

Hoffmann, B., Trinh, T. H., Leung, J., Kondorosi, A., and Kondorosi E. 1997. A new Medicago truncatula line with superior in vitro regeneration, transformation, and symbiotic properties isolated through cell culture selection. Mol. Plant-Microbe Interact. 10:307-315.

Jiménez-Zurdo, J. I., Frugier, F., Crespi, M. D., and Kondorosi, A. 2000. Expression profiles of 22 novel molecular markers for organogenetic pathways acting in alfalfa nodule development. Mol. Plant-Microbe Interact. 13:96-106.

Kaldenhoff, R. and Richter, G. 1989. Sequence of cDNA for a novel light-induced glycine-rich protein. Nucleic Acids Res. 17:2853.

Keller, B., Schmid, J., and Lamb, C. J. 1989. Vascular expression of a bean cell wall glycine-rich protein- $\beta$-glucuronidase gene fusion in transgenic tobacco. EMBO (Eur. Mol. Biol. Organ.) J. 8:1309-1314.

Kingsley, P. D. and Palis, J. 1994. GRP2 proteins contain both CCHC zinc fingers and a cold shock domain. Plant Cell 6:1522-1523.

Kohorn, B. 2001. WAKs: Cell wall associated kinases. Curr. Opin. Cell Biol. 13:529-533.

Kondorosi, E., Banfalvi, Z., and Kondorosi, A. 1984. Physical and genetic analysis of a symbiotic region of Rhizobium meliloti: Identification of nodulation genes. Mol. Gen. Genet. 193:445-452.

Küster, H., Quandt, H. J., Broer, I., Perlick, A. M., and Pühler, A. 1995. The promoter of the Vicia faba L. VfENOD-GRP3 gene encoding a glycine-rich early nodulin mediates a predominant gene expression in the interzone II-III region of transgenic Vicia hirsuta root nodules. Plant Mol. Biol. 29:759-72.

Küster, H., Schröder, G., Frühling, M., Pich, U., Rieping, M., Schubert, I., Perlick, A. M., and Pühler, A. 1995. The nodule-specific VfENODGRP3 gene encoding a glycine-rich early nodulin is located on chromosome I of Vicia faba L. and is predominantly expressed in the interzone II-III of root nodules. Plant Mol. Biol. 28:405-421.

Le, H., Chang, S., Tanguay, R., and Gallie, D. 1997. The wheat poly(A)binding protein functionally complements pab1 in yeast. Eur. J. Biochem. 243:350-357.

Linthorst, H. J., van Loon, L. C., Memelink, J., and Bol, J. F. 1990. Char- 
acterization of cDNA clones for a virus-inducible, glycine-rich protein from petunia. Plant Mol. Biol. 15:671.

Marty, I., Monfort, A., Stiefel, V., Ludevid, D., Delseny, M., and Puigdomenech, P. 1996. Molecular characterization of the gene coding for GPRP, a class of proteins rich in glycine and proline interacting with membranes in Arabidopsis thaliana. Plant Mol. Biol. 30:625-636.

Memelink, J., Linthorst, H. J., Schilperoort, R. A., and Hoge, J. H. 1990. Tobacco genes encoding acidic and basic isoforms of pathogenesis-related proteins display different expression patterns. Plant Mol. Biol. 14:119-126.

Molina, A., Mena, M., Carbonero, P., and García-Olmedo, F. 1997. Differential expression of pathogen-responsive genes encoding two types of glycine-rich proteins in barley. Plant Mol. Biol. 33:803-810.

Mousavi, A., Hiratsuka, R., Takase, H., Hiratsuka, K., and Hotta, Y. 1999. A novel glycine-rich protein is associated with starch grain accumulation during anther development. Plant Cell Physiol. 40:406-416.

Orosz, L., Svab, Z., Kondorosi, A., and Sik, T. 1973. Genetic studies on Rhizobiophage 16-3. I. Genes and functions on the chromosome. Mol. Gen. Genet. 125:341-350.

Park, A. R., Somi, K. C., Yun, U. J., Jin, M. Y., Lee, S. H., SachettoMartins, G., and Park, O. K. 2001. Interaction of the Arabidopsis receptor protein kinase Wak1 with a glycine-rich protein, AtGRP-3. J. Biol. Chem. 276:26688-26693.

Pawlowski, K., Twigg, P., Dobritsa, S., Guan, C., and Mullin, B. C. 1997. A nodule-specific gene family from Alnus glutinosa encodes glycineand histidine-rich proteins expressed in the early stages of actinorhizal nodule development. Mol. Plant-Microbe Interact. 10:656-664.

Potenza, C., Thomas, S. H., and Sengupta-Gopalan, C. 2001. Genes induced during early response to Meloidogyne incognita in roots of resistant and susceptible alfalfa cultivars. Plant Sci. 161:289-299.

Putnoky, P., Grosskopf, E., Ha, D. T., Kiss, G. B., and Kondorosi, A.
1988. Rhizobium fix genes mediate at least two communication steps in symbiotic nodule development. J. Cell Biol. 106:597-607.

Reddy, A. S., and Poovaiah, B. W. 1987. Accumulation of a glycine rich protein in auxin-deprived strawberry fruits. Biochem. Biophys. Res. Commun. 147:885-891.

Rohde, W., Rosch, K., Kroger, K., and Salamini, F. 1990. Nucleotide sequence of a Hordeum vulgare gene encoding a glycine-rich protein with homology to vertebrate cytokeratins. Plant Mol. Biol. 14:10571059.

Ross, J. H., and Murphy, D. J. 1996. Characterization of anther-expressed genes encoding a major class of extracellular oleosin-like proteins in the pollen coat of Brassicaceae. Plant J. 9:625-637.

Sachetto-Martins, G., Franco, L. O., and de Oliveira, D. E. 2000. Plan glycine-rich proteins: A family or just proteins with a common motif? Biochim. Biophys. Acta 1492:1-14.

Santino, C. G., Stanford, G. L., and Conner, T. W. 1997. Developmental and transgenic analysis of two tomato fruit enhanced genes. Plant Mol. Biol. 33:405-416.

Schröder, G., Frühling, M., Pühler, A., and Perlick, A. M. 1997. The temporal and spatial transcription pattern in root nodules of Vicia faba nodulin genes encoding glycine-rich proteins. Plant Mol. Biol. 33:113-123.

Schultze, M., and Kondorosi, A. 1998. Regulation of symbiotic root nodule development. Annu. Rev. Genet. 32:33-57.

Showalter, A. M., Zhou, J., Rumeau, D., Worst, S. G., and Varner, J. E. 1991. Tomato extensin and extensin-like cDNAs: Structure and expression in response to wounding. Plant Mol. Biol. 16:547-565.

von Heijne, G. 1986. A new method for predicting signal sequence cleavage sites. Nucleic Acids Res. 14:4683-4690.

$\mathrm{Xu}, \mathrm{D} ., \mathrm{Lei}, \mathrm{M}$. , and Wu, R. 1995. Expression of the rice Osgrp1 promoter-Gus reporter gene is specifically associated with cell elongation/expansion and differentiation. Plant Mol. Biol. 28:455-471. 